\title{
Surface phase defects induced downstream laser intensity modulation in high-power laser facility
}

\author{
Xin Zhang, Wei Zhou, Wanjun Dai, Dongxia Hu, Xuewei Deng, Wanqing Huang, Lidan Zhou, Qiang Yuan, \\ Xiaoxia Huang, De'en Wang, and Ying Yang \\ Research Center of Laser Fusion, China Academy of Engineering Physics, Mianyang 621900, China \\ (Received 7 November 2015; revised 9 January 2016; accepted 26 January 2016)
}

\begin{abstract}
Optics surface phase defects induced intensity modulation in high-power laser facility for inertial confinement fusion research is studied. Calculations and experiments reveal an exact mapping of the modulation patterns and the optics damage spot distributions from the surface phase defects. Origins are discussed during the processes of optics manufacturing and diagnostics, revealing potential improvements for future optics manufacturing techniques and diagnostic index, which is meaningful for fusion level laser facility construction and its operation safety.
\end{abstract}

Keywords: intensity modulation; optics damage; phase defect

\section{Introduction}

In order to achieve fusion ignition through inertial confinement ${ }^{[1]}$, high-power laser facilities are built to provide enough laser energy and power to compress the fusion fuels in the target pellet, such as the national ignition facility (NIF) in the United States ${ }^{[2]}$, the Laser Megajoule in France ${ }^{[3]}$, and the SG series laser facilities in China ${ }^{[4]}$. Such laser facilities designed for fusion ignition always produce more than $15 \mathrm{~kJ}$ fundamental laser energy in one single beam, which is then tripled to ultraviolet with $10 \mathrm{~kJ}$ laser energy, producing about 12 and $8 \mathrm{~J} / \mathrm{cm}^{2}$ laser fluence in fundamental and ultraviolet sections separately. These relatively high laser fluences impose huge pressures on the optics' resistance to damage, especially in the ultraviolet section ${ }^{[5]}$. In order to overcome such damage threats, technical methods and operation strategies have been developed. On the one hand, the qualities of the optics in ultraviolet section must be improved to suppress the damage initials. For example, NIF spent more than 10 years improving the qualities of the optics and has developed a complicated strategy to circulate the damaged optics ${ }^{[6]}$ in spite of it being costly and inefficient. On the other hand, the beam quality of the fundamental lasers need to be improved to get rid of the optics damage from smallscale intensity modulation, such as the image-relay designs in high-power laser facilities and the improvement of the surface quality of the large-scale optics ${ }^{[7]}$.

Correspondence to: X. Deng, Research Center of Laser Fusion, China Academy of Engineering Physics, Mianyang China, 621900. Email: xwdeng@caep.cn
In this letter, we study surface phase defects induced downstream laser intensity modulation in high-power laser facility. Phase defects that lie on the reflection optics in one of the SG-III laser beamlines are studied. These surface phase defects result in strong intensity modulation after some propagation distance, which is proved harmful to the optics in final optics assembly $(\mathrm{FOA})^{[8,9]}$. Our analysis also reveals that the current diagnostic index for large-scale optics still needs to be improved.

\section{Theoretical analysis}

The principle of phase defects induced laser intensity modulation is well known as shown in Figure 1. If the surfaces of the optics are ideally perfect, then the laser transmitting it or reflected by it will not be affected. However, if there exist some phase defects, they will act as lens, inducing laser focusing or defocusing, which will then evolve into intensity modulation after propagation and cause damage to the downstream optics. In high-power laser system, such phase defects on optics are relatively small and sometimes so tiny that even surface diagnostics cannot reveal their potential threats to downstream optics according to current index. Actually, these tiny phase defects do not induce severe intensity modulation right behind the optics exit surface. But typical laser propagation distance in high-power laser facilities is always several tens of meters, which is long enough to evolve strong small-scale intensity modulation. So such surface phase defects in the fundamental section 


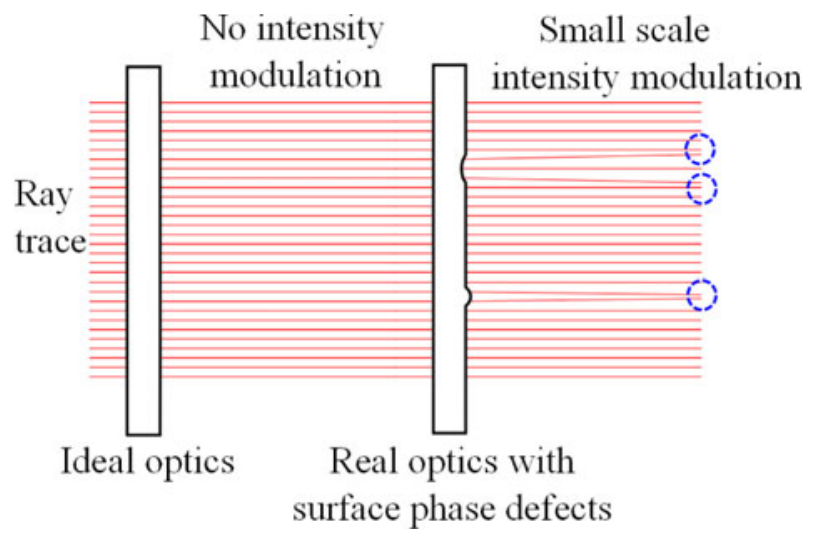

Figure 1. Surface phase defects induced small-scale intensity modulation.

are huge threats to the optics in FOA in the ultraviolet section. Theoretically, such surface phase defects induced intensity modulation after laser propagation can be described by Helmholtz equation,

$$
\nabla^{2} E-\frac{n_{0}^{2}}{c^{2}} \frac{\partial^{2}}{\partial t^{2}} E=0
$$

which can be expressed as Huygens-Fresnel integration under paraxial approximation,

$$
\begin{aligned}
& E_{1}\left(x_{2}, y_{2}\right)=\frac{\exp (i k z)}{i \lambda z} \iint_{-\infty}^{+\infty} E_{1}\left(x_{1}, y_{1}\right) \\
& \quad \times \exp \left\{\frac{i k}{2 z}\left[\left(x_{2}-x_{1}\right)^{2}+\left(y_{2}-y_{1}\right)^{2}\right]\right\} d x_{1} d y_{1} .
\end{aligned}
$$

According to Equation (2), any phase defects induced intensity modulation after laser propagation in high-power laser facilities can be simulated.

\section{Simulation and experiment}

In a general way, online optics in high-power laser facility must pass a series of strict diagnostics before they were installed. Nevertheless, some small defects were artificially neglected according to the current diagnostic index, such as the surface phase defects. For example, we find that there are some phase defects existing on the surfaces of reflecting mirrors in one beamline. As shown in Figure 2, some fringelike phase defects are clearly observed with a modulation depth of about several tens of nanometers. Typically, there are six or seven reflecting mirrors in our laser facility ${ }^{[10]}$, supporting the $360 \mathrm{~mm} \times 360 \mathrm{~mm}$ lasers propagating to the FOA. The observed phase defects exist on the first and the third mirror, respectively. Lasers reflected by the first mirror will propagate over $50 \mathrm{~m}$ to achieve the FOA, during which these defects induced phase modulation will evolve into intensity modulation.
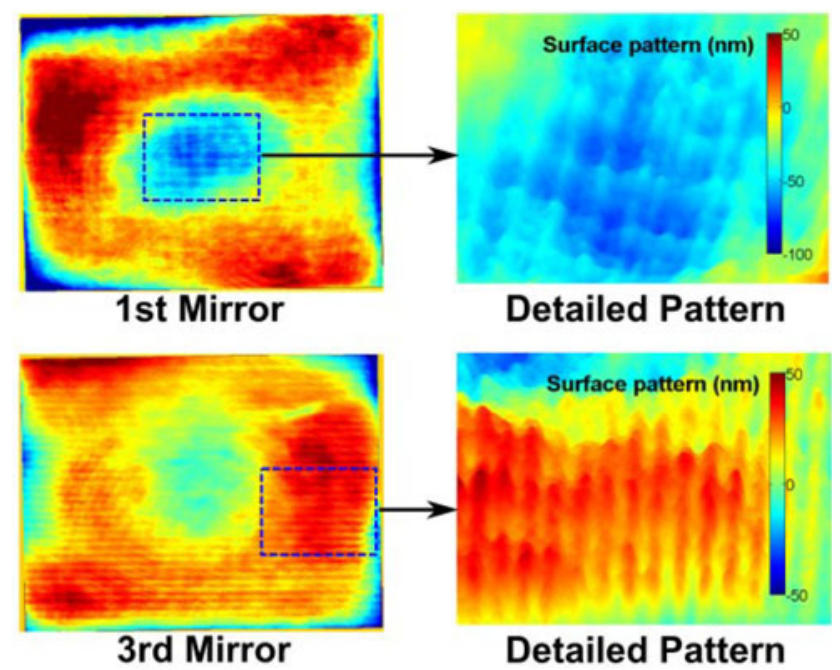

Figure 2. Surface phase defects exist on the reflecting mirrors. These mirrors support lasers with beam size $360 \mathrm{~mm} \times 360 \mathrm{~mm}$.
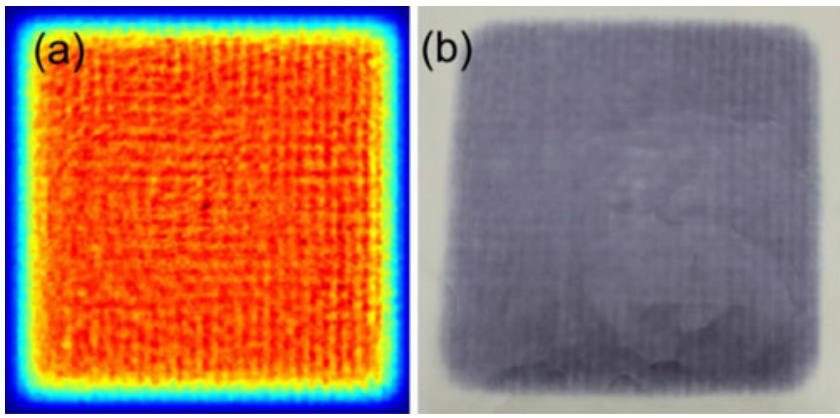

Figure 3. (a) Simulated laser intensity modulation before FOA and (b) the experimental result. The simulated and experimentally captured beam sizes are both $360 \mathrm{~mm} \times 360 \mathrm{~mm}$.

Calculations were made according to Equation (2) to simulate the laser intensity modulation right before the FOA. As shown in Figure 3(a), when an ideal laser with 8thorder-super-Gaussian intensity distribution is reflected by the mirrors with surface phase defects and propagating to the FOA, strong intensity modulation is induced, which looks like an exact mapping of the phase defect patterns on the surfaces of the reflecting mirrors. Experimental demonstration was also made with main shot. The recorded laser intensity distribution is shown in Figure 3(b). Fringe-like intensity modulation is clearly observed in the experiment, which is in perfect accordance with the simulated intensity modulation, demonstrating that surface phase defects on reflecting mirrors will result in severe laser intensity modulation before the FOA.

So much eyesight is focused on laser intensity modulation because it is one of the dominating reasons leading to ultraviolet optics damage. In order to make sure that the facility is operating safely, typical laser intensity modulation 


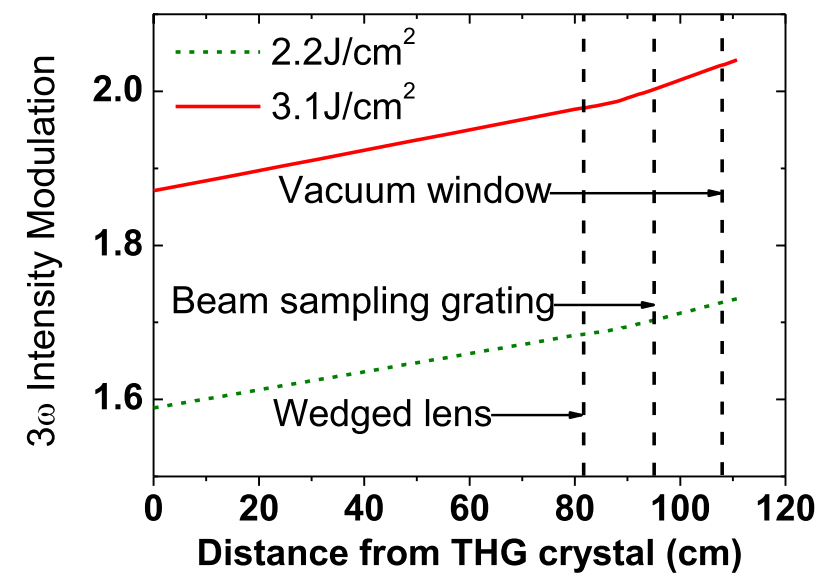

Figure 4. $3 \omega$ intensity modulation in the FOA. THG denotes three harmonic generations.

in the ultraviolet section is carefully kept below 1.8. As a potential threat, laser intensity modulation before the FOA induced by the surface phase defects on the reflecting mirrors is evaluated theoretically. The calculation is made as follows: the nonlinear Schredinger equation is used to solve the laser nonlinear propagation in wedged lens, beam sampling grating and vacuum window; the linear diffraction theory is used to calculate the laser propagation in the air. As shown in Figure 4, $3 \omega$ intensity modulation in the FOA is calculated with $2.2 \mathrm{~J} / \mathrm{cm}^{2}$ (green dashed line) and $3.1 \mathrm{~J} / \mathrm{cm}^{2}$ (red solid line) laser fluences, respectively, which are the common operating laser fluences in our laser facility. When the operating laser fluence is $2.2 \mathrm{~J} / \mathrm{cm}^{2}$, the $3 \omega$ intensity modulation is below 1.8, at which level the surface phase defects bring little threats to the optics in the FOA. While the operating fluence is set to $3.1 \mathrm{~J} / \mathrm{cm}^{2}$, the $3 \omega$ intensity modulation in the FOA exceeds the preset safety line 1.8, imposing huge damage threats onto the last several optics in the FOA, such as the wedged lens, the beam sampling grating and the vacuum window. Obviously, the vacuum window lies at last and endures the highest damage threat.

Experimentally, the vacuum window is diagnosed after main shots with 2.2 and $3.1 \mathrm{~J} / \mathrm{cm}^{2}$ ultraviolet fluences, respectively. After 30 main shots with $2.2 \mathrm{~J} / \mathrm{cm}^{2}$ ultraviolet laser fluence, there were only a few rather small damage spots appearing on the vacuum window, as shown in Figure 5(a). While another nine main shots with $3.1 \mathrm{~J} / \mathrm{cm}^{2}$ ultraviolet fluence was operated, plenty of damage spots appeared as shown in Figure 5(b). The most important information we get from the diagnostic result is that the distribution of these damage spots exactly copy the patterns of the laser intensity modulation induced by the surface phase defects on the reflecting mirrors. Fringe-like damage spot distribution is clearly observed in Figure 5(b) that is the same as the laser intensity modulation in Figure 3, demonstrating the severe damage threat from the mirror surface phase defects onto the ultraviolet optics in the FOA.

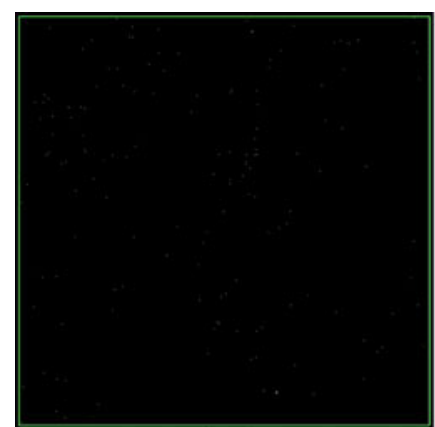

(a)

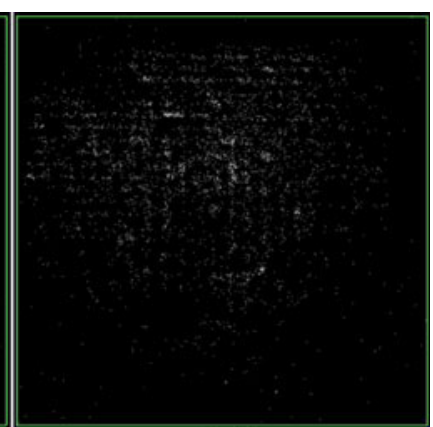

(b)
Figure 5. Damage on the vacuum window after (a) 2.2 and (b) $3.1 \mathrm{~J} / \mathrm{cm}^{2}$ ultraviolet operating fluences. The periphery frame indicates the laserpassing area of $360 \mathrm{~mm} \times 360 \mathrm{~mm}$.

It is quite crucial to discuss the origin of the fringe-like surface phase defects on the reflecting mirrors as well as the current diagnostics index for the optics manufactured for high-power lasers. On the one hand, the fringe-like surface phase defects must come from the manufacturing process of the reflecting mirrors. It is known that at the last polishing stage, a numerically controlled machine with a polishing bistrique of about $8 \mathrm{~mm}$ is used to further suppress the roughness of the mirror surfaces. However, such numerically controlled polishing with small bistrique results in surface modulations as shown in Figure 2. What is worse, these modulations are periodically distributed as optical gratings, which will lead to periodical reappearance of the modulation peaks instead of modulation attenuated by diffraction along the propagation direction due to the Talbot effect ${ }^{[1,12]}$. So it is important to avoid numerically controlled polishing with small bistrique during optics manufacturing. On the other hand, according to current optics diagnostic index, these obvious periodical modulations with depth of several tens of nanometers are not treated as unqualified factors. A primary reason is that during the optics diagnostics, the PV value requirement of the optics surface pattern is always larger than $100 \mathrm{~nm}$, which does not treat the periodical modulations with depth of several tens of nanometers as unqualified defects. As a second reason, during the GRMS value diagnostics of the optics surface pattern, the current index contains a special filtering process that filters out those spatial modulation frequencies higher than $1 / 33 \mathrm{~mm}$. That means the $8 \mathrm{~mm}$ periodical modulation will not be seen during the GRMS diagnostics, nor be treated as unqualified factors.

\section{Conclusion}

In summary, optics surface phase defects induced intensity modulation in high-power laser facility for inertial confinement fusion research is studied. With the increasing of the laser fluence, the intensity modulations impose great 
damage threats to the optics in the FOA. Calculations and experimental results reveal an exact mapping of the laser intensity modulation patterns and the optics damage spot distributions from the phase defects on the optics surface. The origins of these surface phase defects existing in online optics are discussed. Through this study, we find the current optics manufacturing process and the diagnostics index still need to be improved, which is quite important for future construction of fusion level high-power laser facilities and their operation safety.

\section{Acknowledgments}

This work is supported by the SG-III laser facility upgrade project.

\section{References}

1. J. M. Vehn, Plasma Phys. Control. Fusion 39, B39 (1997).

2. C. A. Haynam, P. J. Wegner, J. M. Auerbach, M. W. Bowers, S. N. Dixit, G. V. Erbert, G. M. Heestand, M. A. Henesian,
M. R.Hermann, K. S. Jancaitis, K. R. Manes, C. D. Marshall, N. C. Mehta, J. Menapace, E. Moses, J. R. Murray, M. C. Nostrand, C. D. Orth, R. Patterson, R. A. Sacks, M. J. Shaw, M. Spaeth, S. B. Sutton, W. H. Williams, C. C. Widmayer, R. K. White, S. T. Yang, and B. M. Van Wonterghem, Appl. Opt. 46, 3276 (2007).

3. M. L. Andre, Proc. SPIE 3047, 38 (1997).

4. X. Deng, Q. Zhu, W. Zheng, X. Wei, F. Jing, D. Hu, W. Zhou, B. Feng, J. Wang, Z. Peng, L. Liu, Y. Chen, L. Ding, D. Lin, L. Guo, and Z. Dang, Proc. SPIE 9266, 926607 (2014).

5. W. Han, W. Huang, F. Wang, K. Li, B. Feng, F. Li, F. Jing, and W. Zheng, Chin. Phys. B 19, 106105 (2010).

6. S. Papernov and A. W. Schmid, Proc. SPIE 7132, 71321J (2008).

7. L. Wong, T. Suratwala, M. D. Feit, P. E. Miller, and R. Steele, J. Non-Cryst. Solids 355, 797 (2009).

8. L. S. Dauffy, H. Y. Khater, and S. Sitaraman, Fusion Sci. Technol. 56, 736 (2009).

9. L. Parietti and R. A. Martin, FED (Am. Soc. Mech. Eng.) 245, 3/76 (1998)

10. X. Deng, F. Wang, H. Jia, Y. Xiang, B. Feng, K. Li, and L. Zhou, Chin. Phys. Lett. 29, 124211 (2012).

11. H. Hamam, Appl. Opt. 43, 7052 (2003).

12. S. Teng, L. Liu, and J. Zu, J. Opt. Soc. Am. A 20, 1747 (2003). 